Paper

\title{
Touchscreen Text Entry Methods Used by Blind and Low Vision Users
}

Analysis of ICT User Survey 2017

\author{
Tetsuya Watanabe ${ }^{\dagger 1}$, Hirotsugu Kaga ${ }^{\dagger 2}$, Makoto Kobayashi ${ }^{\dagger 3}$, \\ Kazunori Minatani ${ }^{\dagger 4}$
}

\begin{abstract}
A survey was conducted on the use of ICT devices by blind and visually impaired people. Of the 303 respondents, 180 (59.4\%) used feature phones (keitai), 161 (53.1\%) used smartphones, $64(21.1 \%)$ used tablets, and $285(94.1 \%)$ used personal computers. The usage rate of smartphones doubled, and that of feature phones decreased by about $20 \%$. The higher the age was, the higher the usage rate of feature phones and the lower that of smartphones became. The text entry methods on touchscreens with a screen reader varied greatly between blind and low vision people and between smartphones and tablets. Vision aid apps such as for GPS navigation and object recognition were used by more than half of the blind smartphone users.
\end{abstract}

Key words: Blind, Low Vision, Smartphone, Tablet, User Survey, Text Entry.

\section{Introduction}

Information and Communication Technology (ICT) devices have enabled blind and visually impaired people to obtain and communicate information by themselves. With these devices, users can read a variety of books and documents whenever they like without relying on sighted persons, whereas traditional human support such as braille description and audio recording takes a month or more for transformation to be completed $^{11}$.

Traditionally, personal computers ( $\mathrm{PCs}$ ) have been playing a leading role in providing blind and visually impaired people with access to mainly text information. Recently, however, smartphones and tablets are taking over this role ${ }^{2)}$. They not only give access to text information and the Internet but also have functions and apps for speech recognition, image recognition, GPS navigation, and others that enable blind and visually impaired people to live high-quality, independent lives. As these devices are handy, blind and visually impaired people can utilize them whenever and wherever they

Received October 24, 2018; Revised April 26, 2019; Accepted May 8, 2019

$\dagger 1$ Faculty of Engineering, Niigata University

(Niigata, Japan)

$\dagger 2$ Graduate School of Science and Technology, Niigata University. (Niigata, Japan)

$\dagger 3$ Faculty of Health Sciences, Tsukuba University of Technology

(Tsukuba, Japan)

$\dagger 4$ National Center for University Entrance Examinations

(Meguro-ku, Tokyo, Japan) want to. However, blind users have difficulty operating touch interfaces since these devices have no tactile cues $^{2}$. Thus, not only blind and visually impaired people and their supporters but also other stakeholders such as smartphone and tablet makers, marketers, carriers, and administrative officers are showing an interest in the usage of these devices. With this background, we decided to carry out a new survey on the use of ICT devices by blind and visually impaired people. The following is what we wish to make clear out of this survey.

- Latest usage rate of smartphones and tablets

- Age-related differences in usage rate

- Usage differences between blind and low vision

- Latest share of each device

- Text entry methods on touchscreens

- Usage rate of vision aid apps

Among these findings, this paper focuses on text entry methods on touchscreens since this was the biggest issue for blind and visually impaired users in our previous survey ${ }^{2)}$.

\section{Related Surveys}

The first ICT blind user survey in Japan that focused on smartphones and tablets may be the one conducted by Matsuzaka from November 2011 to February $2012^{3)}$. The same research group carried out two more surveys in 2012 and 2013 to explore how touchscreens were used by blind people ${ }^{4}$. Nikkei BP Consulting Inc. carried out a survey on the use of the Internet by blind and visu- 
ally impaired people from October to November $2014^{5)}$. The results showed how blind and visually impaired people used the Internet by means of PCs and smartphones. The authors had previously conducted four ICT user surveys on visually impaired people, one each in 2000, 2002, 2007, and 2013. Among them, the 2013 survey revealed the usage rate and the advantages and drawbacks of smartphones and tablets statistically ${ }^{2}$.

Globally, WebAIM (Accessibility In Mind) has done seven screen reader user surveys from 2008 to $2017^{6)}$. The high frequency of survey conduction and the variety of respondents from all over the world are remarkable.

\section{Survey Procedure}

The survey was contracted to a non-profit organization "Turtle" whose main activity is to help visually impaired people in seeking and keeping their careers $^{7)}$. They disseminated the questionnaire to about 50 mailing lists to which visually impaired people belonged. The respondents answered to the questionnaire by writing a reply message and sending it to the Turtle. For descriptive questions, they wrote the answers in the next line to the question or in the bracket following the question, and for closed-end questions by placing an arbitrary symbol such as a circle in front of the choice they chose. The survey started on February 20, 2017 and ended on March 20, 2017.

The questionnaire first asked about the respondents' profiles and whether they used feature phones, smartphones, tablets, and PCs. If they used one of them, then they were instructed to move on to the following parts where they were asked to describe the model(s) and accessibility features in use, purposes of the use, Web sites they browsed from each device, conveniences, and inconveniences they faced while using them. As for smartphones and tablets, text entry methods and learning methods were asked about in addition. The number of questions on respondents' profiles was five, three on the usage of ICT devices in general, seven on feature phone usage, and nine on each of smartphone, tablet, and PC usage along with a few sub-questions. Most of the questions in the current survey were the same as those in the previous survey except for the detailed questions as to text entry methods.

This survey was reviewed by the Ethics Committee of Niigata University and conducted with the permission of the President (permission number: 2016-0026).

\section{Respondents}

The total number of valid respondents was 303 . This number was smaller than that of the 2013 user survey by just one ${ }^{2)}$. As the mailing lists in which the questionnaire was circulated were mostly the same as in the previous survey in 2013 , it is presumable that the respondents in 2017 survey may overlap those in 2013 greatly. However, we cannot confirm it because the personal information of the respondents such as name, address, and e-mail address was removed before we receive the responses. The 303 respondents comprised 190 men $(62.7 \%)$ and 113 women $(37.3 \%)$ ranging in age groups from teens to 80 years or older (Fig. 1). Those in their fifties were the biggest age group with 76 respondents (25.1\%) followed by the forties (65 respondents, $21.5 \%$ ) and the sixties (54 respondents, 17.8\%).

Two hundred and seven $(68.3 \%)$ were registered as grade 1 visually impaired persons, 68 (22.4\%) as grade 2 , and $23(7.6 \%)$ as grades 3 through 6 . Four persons were not registered as visually impaired, and the status of one was unknown. The Japanese Law for the Welfare of Physically Disabled Persons classifies visually impaired people into six grades and people with grades 1 and 2 disabilities are considered severely impaired.

Among grade 1 respondents, $92.3 \%$ stated that they could not read nor write print. This ratio declined as the degree of disability decreased, $32.4 \%$ for grade 2 and $0 \%$ for grades 3 through 6 (Fig. 2). A survey conducted by a research group in University of Tsukuba on the students attending schools for the blind has shown the relationship between their visual acuity and the letters they use: When their visual acuity is 0.02 or less than that, the usage rate of normal (printed or written) letters declined sharply and that of Braille rose accordingly ${ }^{8)}$. Persons whose visual acuity of both eyes is 0.02 to 0.04 are classified as disability grade 2 . Thus, it is reasonable that the grade 2 respondents in our survey included both people who could read and write print and people who could not. Hereafter, we refer to the respondents who could not read nor write print "blind people" and those who could read and write as "people with low vision." The survey data were categorized into these two user groups and compared with each other.

\section{Usage of ICT Devices in General}

Among the 303 respondents, 180 (54.9\%) used feature phones, 161 (53.1\%) smartphones, 64 (21.1\%) tablets, and $285(94.1 \%)$ PCs. 


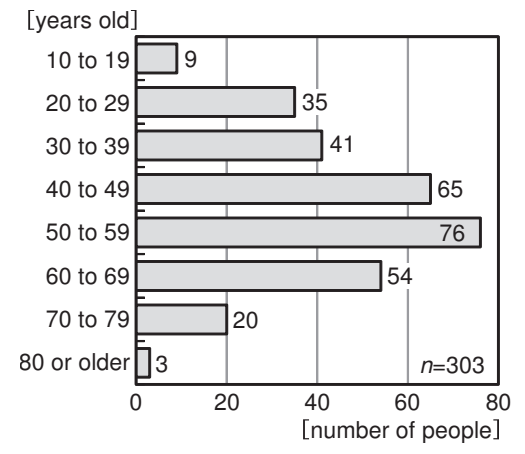

Fig. 1 Age distribution of respondents.

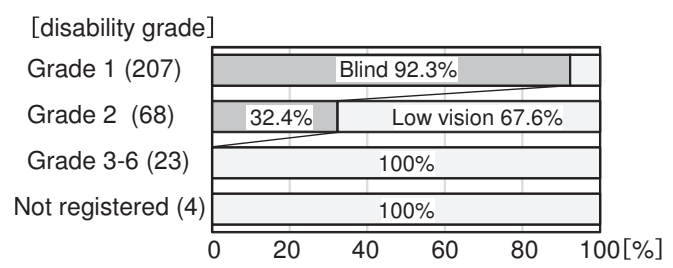

Fig. 2 Ratio of blind to low vision respondents by disability grade.

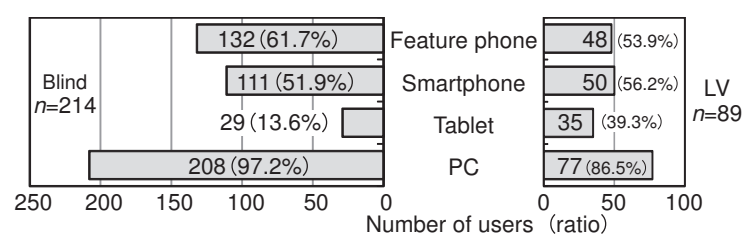

Fig. 3 Usage of ICT devices.

\section{1 Usage Difference between Blind and Low Vision}

The usage rate data were classified in two user groups: blind and low vision (Fig. 3). Four $\chi^{2}$ tests showed no significant difference between blind and low vision people in the usage rate of feature phones and smartphones $\left(\chi^{2}(1)=1.57\right.$ and 0.47 , respectively $)$, whereas significant differences were found in the use of tablets and PCs $\left(\chi^{2}(1)=25.1\right.$ and 12.8, $p<0.05$, respectively). The same $p$ value was used hereinafter.

When compared with the 2013 survey results ${ }^{2}$, the usage rate of smartphones doubled for both blind and low vision people. The usage rate of tablets doubled for low vision people but grew by only 1.4 times for blind people. In contrast, the usage rate of feature phones decreased by $20 \%$ for both blind and low vision people. The usage rate of PCs by blind people kept as high as nearly $100 \%$, whereas that by low vision people dropped by $7.4 \%$.

\subsection{Usage Difference by Age Group}

Prior to the investigation of age difference, the difference in the ratio of blind to low vision people was tested among age groups. From teens to the seventies, the ratio of blind people ranged from $55.6 \%$ to $77.1 \%$. A $\chi^{2}$ test showed no significant difference in the ratio of blind to low vision people among age groups $\left(\chi^{2}(6)=4.54\right.$. Since the size of the age group of 80 s and older was as small as three people, it was excluded from the test.). Thus, in the investigation of age difference, the data of both blind and low vision people were summed up by age group.

Figures 4 (a) to (d) show the usage rate of four types of ICT devices by age group. Apparently, the older the respondents were, the higher the usage rate of feature phones became (Fig 4 (a)). In contrast, the younger the respondents were, the higher the usage rate of smartphones became (Fig 4 (b)). Age group differences were not noticeable for tablets and PCs. Statistical analysis proved these phenomena. Four $\chi^{2}$ tests showed significant differences by age group in the usage rate of feature phones and smartphones $\left(\chi^{2}(6)=47.1\right.$ and 48.4, respectively), whereas no significant age difference was found in the use of tablets and PCs $\left(\chi^{2}(6)=5.25\right.$ and 6.40 , respectively).

\section{Smartphone Usage}

This chapter describes the responses from 161 (111 blind and 50 low vision) users to questions on smartphone usage.

\section{1 Models}

The smartphone models being used by the respondents are shown in Fig. 5. The most popular models were various Apple iPhones with 102 (91.9\% of the 111) blind and 40 ( $80.0 \%$ of the 50$)$ low vision users. "RakuRaku (easy to use) Smartphones" from Fujitsu, which are equipped with a speech output function, were used by only six blind respondents, a very small number compared with the 102 for iPhones. Android devices were used by 14 respondents and 9 of them were people with low vision. Five respondents listed two devices or more, two respondent's devices were not identified, and one did not answer this question. Thus, the summed up numbers in Fig. 5 do not equal the number of total users.

\section{2 Accessibility Features}

Usage rates of accessibility features differed greatly between blind and low vision users (Fig. 6). Most (99.1\%) of the 111 blind smartphone users used speech output (screen reader), and only $7(6.3 \%)$ used visual aids. In contrast, most (90.0\%) of the low vision users used visual accessibility features such as Large Text (70.0\%), Zoom (68.0\%), and Invert Colors (40.0\%). It is worth noting that 24 low vision users $(48.0 \%)$ 


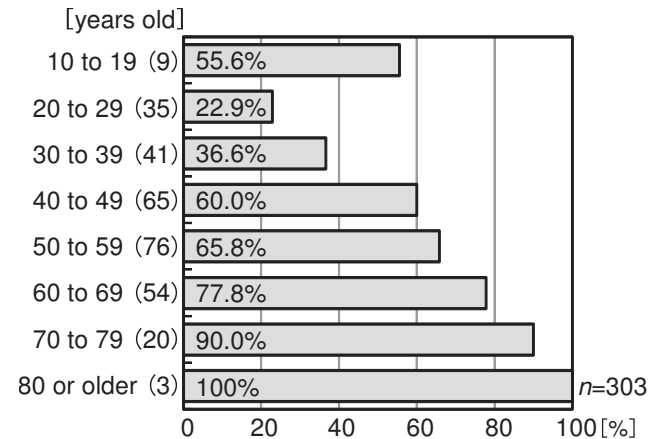

(a) Usage rate of feature phones.

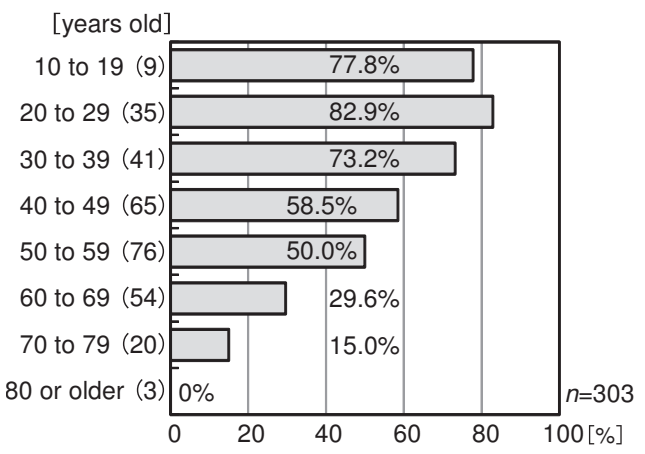

(b) Usage rate of smartphones.

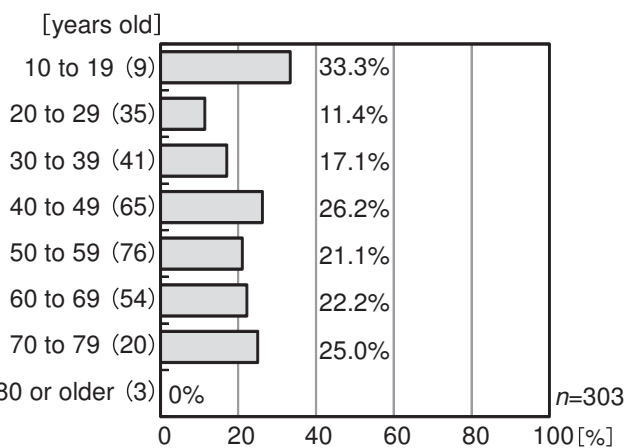

(c) Usage rate of tablets.

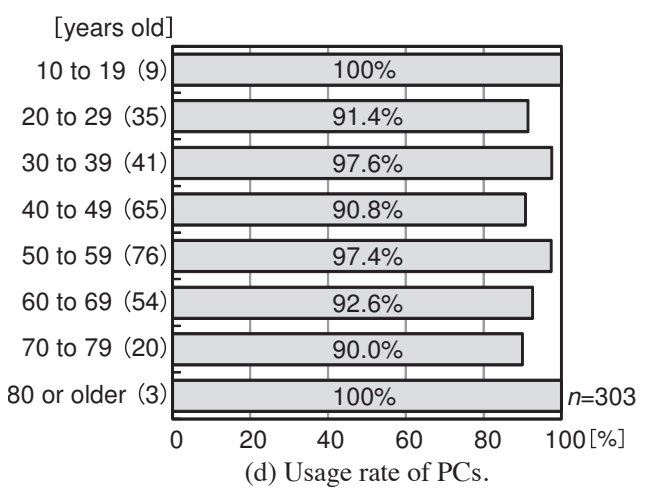

Fig. 4 Usage rate by age group.

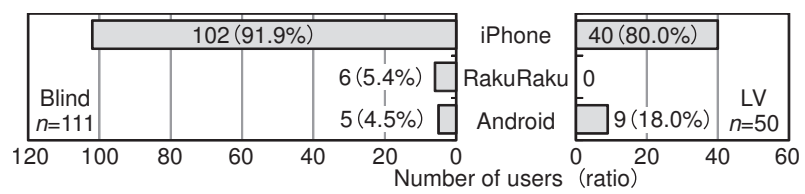

Fig. 5 Popular smartphone models.

used speech output along with visual aids (21 users) or speech output only (3 users) (Fig. 7).

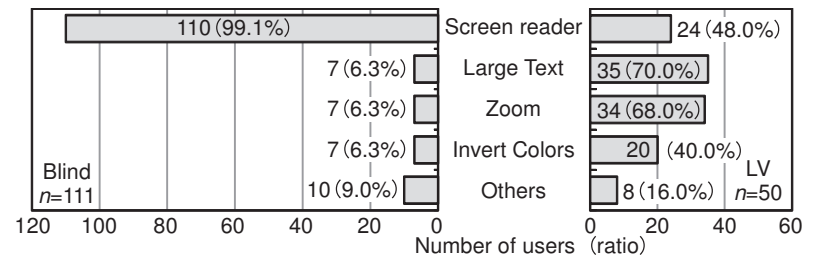

Fig. 6 Accessibility features used on smartphones.

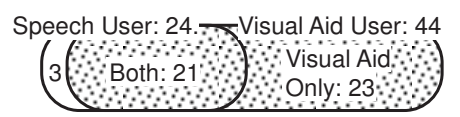

Fig. 7 Overlapping of accessibility feature usage on smartphones.

\section{3 Text Entry Methods}

The questions on the text entry methods on smartphones were in three parts. First, respondents were asked which hardware or software they were using. The result was that software keyboards were used by about $90 \%$ of both blind and low vision users. As for blind users, voice input and hardware keyboards also had high usage rates, $53.2 \%$ and $35.1 \%$, respectively (Fig. 8).

Second, the keyboard users were asked about the type of keyboard. Among 95 blind users of software keyboards, more than half were using the QWERTY keyboard and Japanese numeric keypad (61.1\% and $55.8 \%$, respectively) (Fig. 9, 11). In contrast, among the 45 low vision users of software keyboards, $75.6 \%$ were using the Japanese numeric keypad, and the usage rate of the QWERTY keyboard was as low as $40.0 \%$. The usage rates of the Hiragana keyboard were low for both blind and low vision users. This could be because of the small key pitch (the distance between the centers of two neighboring keys) of the keyboard, which has 50 keys, nearly twice that of the QWERTY keyboard.

When a screen reader is turned on, touch gestures for selecting and entering text change from the norm. In normal mode, a single-tap gesture enters a character. However, in screen reading mode, a single tap gesture has the role of making a touched key a candidate and saying that key aloud. To enter that key, a "double-tap" or "split-tap" gesture is required. Double-tap involves tapping one key two times quickly. Split-tap involves holding a key with one finger and tapping the screen with another finger. Among 110 blind users of screen readers, the number of split-tap users was the topmost (49 users, 44.5\%), and that of double-tap users was the second most (45 users, 40.9\%). However, their difference was small. The number of users of touch input 


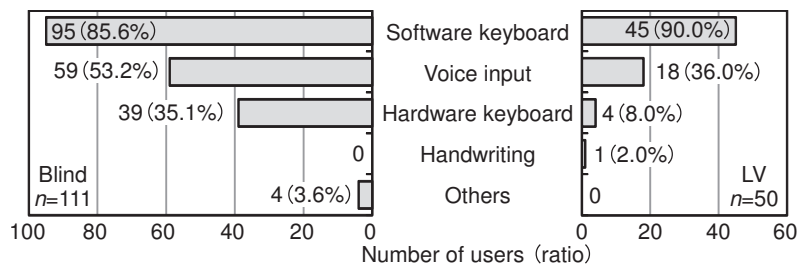

Fig. 8 Text entry methods used on smartphones.

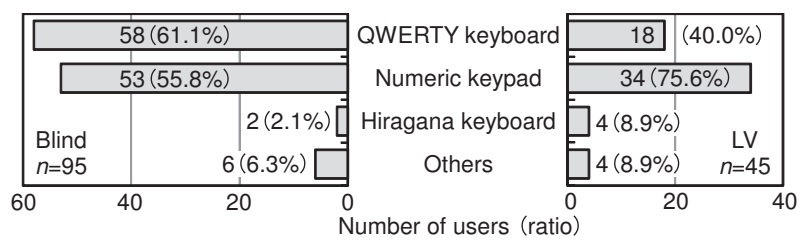

Fig. 9 Software keyboards used on smartphones.

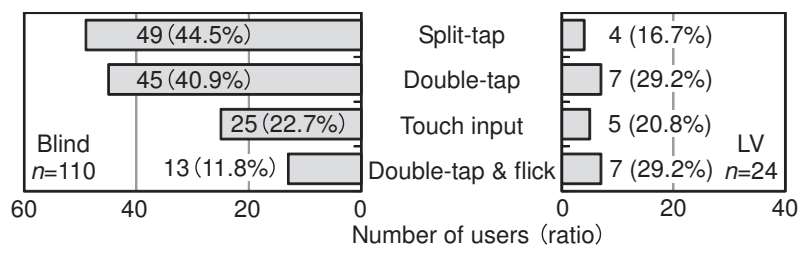

Fig. 10 Character selection gestures used on smartphones.

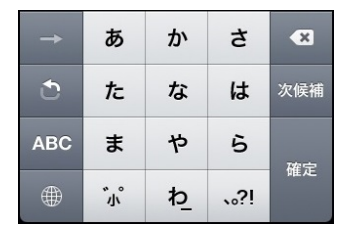

Fig. 11 Japanese numeric keypad.

mode was half of that of split-tap users, and the number of users of double-tap \& flick was half of touch input users. Since the number of low vision users of screen readers was as small as 24, a comparison of the users of each gesture for selecting characters was not meaningful.

The low vision users who did not use a screen reader used the flick input (20 users, $76.9 \%$ of 26 non screen reader users) and toggle input methods (11 users, $42.3 \%)$.

\section{4 Apps}

We presented 26 kinds of apps and had the respondents select and check the apps they were using and write down the names of the apps if possible. In this subsection, we focus on the use of four kinds of vision aid apps that were originally developed for blind and visually impaired people and two standard apps, camera and photo viewer, as they are quite useful for low vision users as vision aids.

Out of the four kinds of apps, GPS and object identifier apps were used by more than half of blind smart-

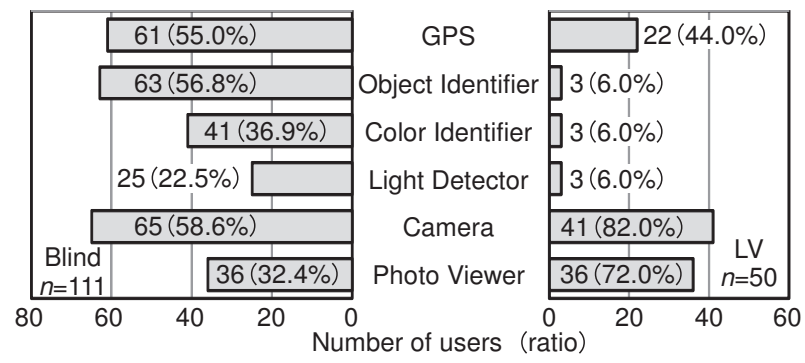

Fig. 12 Vision aid apps used on smartphones.

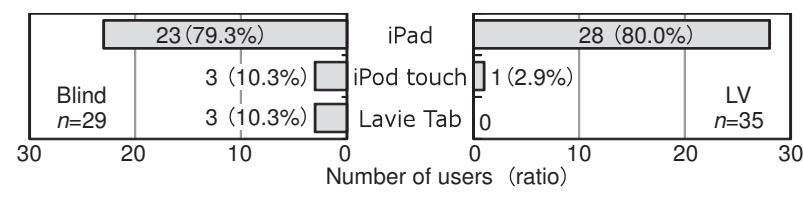

Fig. 13 Popular tablet models.

phone users (GPS apps were used by 61 users (55.0\%) and object identifier apps by 63 users (56.8\%), Fig. 12). Color identifier and light detector apps had fewer users than these. Despite this, it was confirmed that they were used by blind people. Only GPS apps were used by many low vision users (22 users, $44.0 \%$ ).

Camera and photo viewer apps were used by most of the low vision users of smartphones (camera was used by 41 users $(82.0 \%)$ and photo viewer by 36 users $(72.0 \%))$. Although camera apps were used by more than half of the blind users of smartphones (65 users, $58.6 \%$ ), photo viewer apps were used by only one-third of them (36 users, $32.4 \%$ ).

\section{Tablet Usage}

This chapter describes the responses from 64 (29 blind and 35 low vision) users to questions on tablet usage.

\subsection{Models}

The tablet models being used by the respondents are shown in Fig. 13. The most popular models were various Apple iPads with 23 (79.3\% of the 29$)$ blind and $28(80.0 \%$ of the 35$)$ low vision users. Other devices used by more than one respondent were iPod touch and NEC Lavie Tab. Five respondents listed two devices or more, and one did not answer this question. Thus, the summed up number in Fig. 13 does not equal the number of total users.

\subsection{Accessibility Features}

Regarding the usage of tablets, the usage rates for accessibility features differed greatly between blind and low vision users too (Fig.14). The shapes of the graph in Fig. 14 bore a close resemblance to those in Fig. 6. 


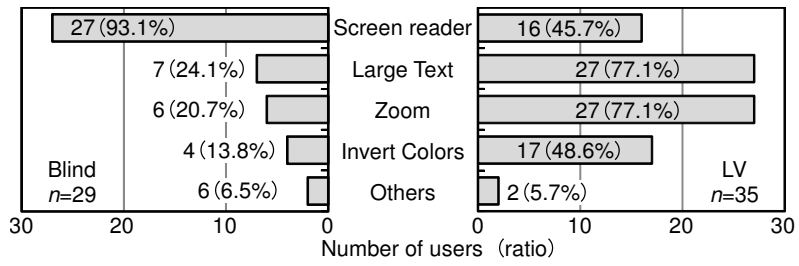

Fig. 14 Accessibility features used on tablets.

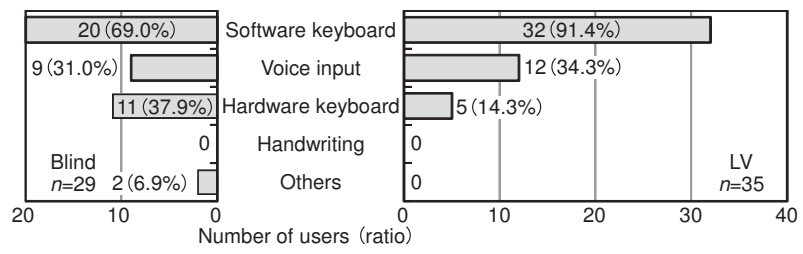

Fig. 15 Text entry methods used on tablets.

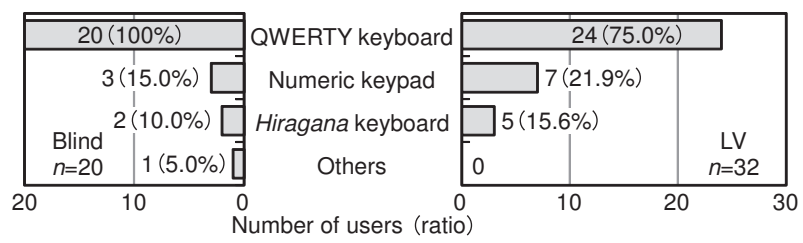

Fig. 16 Software keyboards used on tablets.

\subsection{Text Entry Methods}

The questions on the text entry methods on tablets were the same as those on smartphones. For both blind and low vision users, software keyboards, voice input, and hardware keyboards were the top three text entry methods on tablets (Fig. 15). The usage rates of these methods for low vision users were close to those on smartphones. In contrast, the usage rates of software keyboards and voice input for blind users were roughly $20 \%$ lower than those on smartphones (Fig. 8). This means that fewer blind people were using multiple text entry methods on tablets than on smartphones. No one used handwriting on tablets. Two blind people used "touch braille input" of PC-Talker, a Windows screen reader.

For both blind and low vision users, the QWERTY keyboard was the most used keyboard on tablets with 20 blind users (100\%) and 24 low vision users (75.0\%) (Fig. 16). A striking difference from smartphones was the low usage rates of the numeric keypad for both blind and low vision users.

The touch gesture for selecting characters that was used by the most blind users on tablets was double-tap (15 users, 55\%) (Fig. 7), different from that on smartphones (split-tap, Fig. 10). Among low vision users, touch input was the most common gesture for selsecting characters (8 users, $50.0 \%$ ).

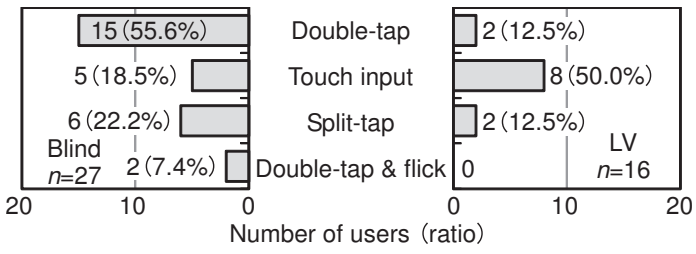

Fig. 17 Character selection gestures used on tablets.

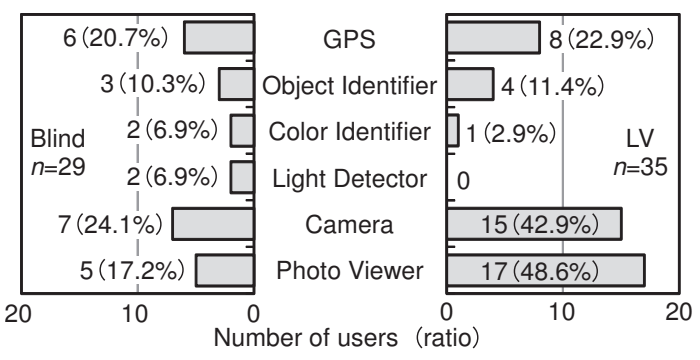

Fig. 18 Vision aid apps used on tablets.

\subsection{Apps}

The usage rates of the six apps that were introduced in the chapter on smartphone usage are shown in Fig. 18. Camera and photo viewer apps were used by nearly half of low vision users $(15(42.9 \%)$ and 17 users $(48.6 \%)$, respectively). However, the number of users of the four vision aid apps was low, less than nine users. The numbers of blind users who used the four apps and camera and photo viewer apps were as low as seven or less.

\section{Discussion}

On the basis of the survey results, let us discuss the questions posed in Introduction.

\section{1 Device Usage Rate}

The latest usage rate of smartphones demonstrated an increase in smartphone and decrease in feature phone usage rates from 2013 to 2017. These phenomena were not peculiar to the lives of blind and visually impaired people but were the same for general (sighted) people. As for the mobile phone usage of sighted persons, NTT docomo's starting to handle the iPhone was the biggest reason for the turnaround in the usage rates of smartphones and feature phones ${ }^{9}$. As for blind and visually impaired people, the September 2014 release of iOS 6, which, for the first time on smartphones, adopted Shosaiyomi, which are Kanji explanatory expressions ${ }^{10)}$, pushed up the usage rate of smartphones. Our two surveys in 2013 and 2017 accurately observed this transition from feature phones to smartphones.

\section{2 Age-Related Differences in Usage Rate}

High usage rates of smartphones among younger generations and those of feature phones among older gener- 
ations were observed. These phenomena were also the same for sighted people ${ }^{11)}$.

Similarly, the phenomenon that the younger the users are, the higher the usage rate of new devices had been observed when feature phones (called as mobile phones at that time) prevailed among visually impaired peo$\mathrm{ple}^{12)}$.

\subsection{Usage Differences between Blind and Low Vision}

Significant differences were found in the use of tablets and PCs between blind and low vision. As for tablet usage, it is because tablets are useful for people with low vision as vision aid (see Fig.18) whereas tablets are too large to use for blind people ${ }^{13)}$.

\section{4 Latest Device Shares}

For both smartphone and tablet usage, devices from Apple Inc. were the most popular. This is because the accessibility features in $\mathrm{iOS}$ are ample and satisfactory for blind and visually impaired people ${ }^{14)}$. If there is a certain number of users of specific products, the convenience of being able to ask questions on the use of these products would lead to an additional increase in the number of users of these products.

The same phenomenon had been observed when the "Raku-Raku Phones" series feature phones from Fujitsu that had special accessibility features such as voice output and large letters were dominant among visually impaired users ${ }^{12)}$.

\section{5 Text Entry Methods on Touchscreen}

Text entry on touchscreens without tactile cues is the biggest challenge for blind and visually impaired peo$\mathrm{ple}^{2)}$. The present survey made clear how they were dealing with this challenge in detail; text entry methods on touchscreens varied greatly between blind and low vision people and between smartphones and tablets. Most of the low vision smartphone users were using Japanese numeric software keyboards. This usage is identical to that of sighted people. In contrast, high usage rates of voice input and hardware keyboards by blind smartphone users were noticeable. These users mainly used QWERTY and numeric keyboards at the same rate. The usage rates of each gesture for selecting characters when using screen readers were determined for the first time.

In the meantime, we are carrying out an experiment in which text entry speeds with a screen reader on a smartphone are being measured with various keyboards and character selection methods as conditions ${ }^{15)}$. The results of this experiment as well as the present survey
Table 1 Key pitches of different keyboards on smartphone (iPhone 6) and tablet (iPad mini).

\begin{tabular}{|c|c||c|c|}
\hline \hline \multirow{2}{*}{ Keyboard } & \multirow{2}{*}{ Direction } & \multicolumn{2}{|c|}{ Key pitch [mm] } \\
\cline { 3 - 4 } & & Smartphone & Tablet \\
\hline \hline \multirow{2}{*}{$\begin{array}{c}\text { Japanese } \\
\text { numeric }\end{array}$} & Horizontal & 11.5 & 9.8 \\
\cline { 2 - 4 } & Vertical & 8.3 & 8.4 \\
\hline QWERTY & Horizontal & 5.85 & 10.7 \\
\cline { 2 - 4 } & Vertical & 8.45 & 10.25 \\
\hline Hiragana & Horizontal & - & 8.55 \\
\cline { 2 - 4 } & Vertical & - & 8.0 \\
\hline \hline
\end{tabular}

results would be very useful for ICT instructors who teach blind people how to use smartphones.

On tablets, QWERTY was the most-used keyboard for both blind and low vision users. This would be because the wider key pitches than on smartphones allow users to select keys precisely. The horizontal and vertical key pitches of the Japanese numeric keypad, QWERTY keyboard, and Hiragana keyboard (only for tablets) on the most popular smartphone model, iPhone 6 , and on the most popular tablet model, iPad mini, are shown in Table 1. It was interesting that the character selection gestures used by more than half of the blind users were different from those on smartphones. How the size of a device changes the character selection gestures to be used is worth being investigated in the future.

\section{6 Usage Rate of Vision Aid Apps}

GPS navigation and object recognition apps were used by more than half of the blind smartphone users. In addition to this, color recognition and light detection apps were used by about one-third and one-fifth of the blind smartphone users. These figures show the usefulness of these vision aid apps for blind people. However, fewer blind people used these apps on tablets. This suggests the superiority of smartphones in terms of portability and usability over tablets. In comparison, the numbers of low vision users of these vision aid apps were quite low except for GPS navigation. Instead, nearly half of low vision smartphone users used camera and picture apps. Some functions of these apps would be playing the role of zooming and color-inverting vision aids.

\section{Conclusion}

An ICT user survey was conducted to investigate usage by blind and low vision people. The findings can be utilized as a basis for developing new easier-to-use text entry methods, vision aid apps, and mobile devices and for designing new accessibility policies. 


\section{Acknowledgments}

We deeply appreciate Mr. H. Matsuzaka and H. Nakamoto of NPO Turtle for conducting the survey, C. Saito for entering the 303 responses into a spreadsheet, and the 303 respondents for answering the long questionnaire. This work was supported by the Ministry of Health, Labor and Welfare Grants-in-aid for Scientific Research (H28-Physical and Intellectual-General-010).

\section{References}

1) T. Watanabe, M. Kobayashi, and K. Minatani: "A User Survey on Braille Transcription and Audio Recording Services for the Blind," Trans. of Human Interface Society, 20, 1, pp.13-20 (Feb. 2018)

2) T. Watanabe, T. Yamaguchi, and K. Minatani: "Advantages and Drawbacks of Smartphones and Tablets to Visually Impaired People - Analysis of the Results of the ICT User Survey -," IEICE Trans. on Information \& Systems, E98-D, 4, pp.922-929 (April 2015)

3) H. Matsuzaka: "A Study on the Use of Touchscreen Devices by Blind and Visually Impaired People and its User Interface," Master's thesis, Tsukuba University of Technology (2013)

4) T. Miura, M. Sakajiri, H. Matsuzaka, M. Eljailani, K. Kudo, N. Kitamura, J. Onishi, T. Ono: "Usage Situation Changes of Touchscreen Computers in Japanese Visually Impaired People: Questionnaire Surveys in 2011-2013," Proc. ICCHP 2014: Computers Helping People with Special Needs, pp.360-368 (July 2014)

5) Nikkei BP Consulting, Inc.: "Internet User Survey by People with Disabilities 2015 (Blind and Visually Impaired People)," https://consult.nikkeibp.co.jp/info/news/2015/0128sa-2/ (2015)

6) WebAIM: "Survey of Preferences of Screen Readers Users," https://webaim.org/projects/screenreadersurvey/ (2018)

7) NPO Turtle: http://www.turtle.gr.jp/ (2018)

8) T. Kakizawa, K. Kagawa, Y. Toriyama, H. Nakata, N. Iketani, T. Sashima: "A 2000 Survey of Students in Special Schools for the Visually Impaired in Japan," Bull. Special Education, 26, pp.163-175 (2002)

9) JustSystem : "Latest Smartphone Penetration Rate in 2016 by sex, area, and age," https://marketing-rc.com/article/20160731.html (2016)

10) T. Watanabe, N. Osugi, T. Yamaguchi, B. Watanabe, S. Okada, and M. Sawada: "Development and Evaluation of Kanji Explanatory Expressions Based on Vocabulary Characteristics of School Children -Improvement of Shosaiyomi of Screen Readers for Blind Persons-," IEICE Trans. on Information \& Systems, J90-D, 6, pp.1521-1531 (June 2007)

11) Ministry of Internal Affairs and Communications : "Penetration of Smartphones and Usage of ICT," 2016 White Paper on Information and Communication in Japan (PDF version), http://www.soumu.go.jp/johotsusintokei/whitepaper/ja/h28/pdf/ (2017)

12) T. Watanabe, M. Miyagi, K. Minatani, H. Nagaoka: "A Survey on the Use of Mobile Phones by Visually Impaired Persons in Japan," Proc. ICCHP 2008: Computers Helping People with Special Needs, pp.1081-1084 (July 2008)

13) R. Yamazaki, T. Watanabe: "Effects of Touchscreen Device Size on Non-Visual Icon Search," IEICE Trans. on Information \& Systems, E100-D, 12, pp.3050-3053 (Dec. 2017)

14) Apple Inc. : "Vision Accessibility," https://www.apple.com/jp/ accessibility/iphone/vision/ (2018)

15) T. Watanabe, H. Kaga and S. Shinkai: "Comparison of Onscreen Text Entry Methods when Using a Screen Reader," IEICE Trans. on Information \& Systems, E101-D, 2, pp.455-461 (Feb. 2018)

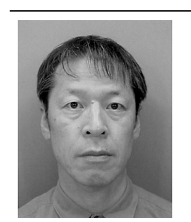

Tetsuya Watanabe received a M.S. degree in Biomedical Engineering in 1993 and a Doctor's degree in Information System Engineering in 2001, both from Hokkaido University. He was at the National Institute of Vocational Rehabilitation from 1994 to 2001 and the National Institute of Special Needs Education from 2001 to 2009. He is now an Associate Professor at Niigata University and doing research in assistive technology for visually impaired people.

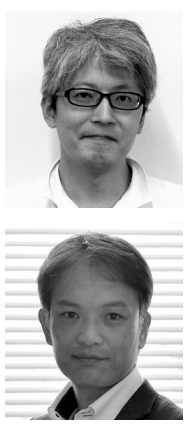

Hirotsugu Kaga received an M.B.A. from Shukutoku University in 2002 and a Doctor's degree in Engineering in 2019 from Niigata University. His current research topic is assistive technology for visually impaired people.

Makoto Kobayashi received a M.S. degree in Engineering in 1995 and a Doctor's degree in Engineering in 2002, both from the University of Tsukuba. He is an Associate Professor in the Department of Computer Science, Tsukuba University of Technology. His research interests include systems for supporting visually impaired or hearing impaired people in their daily lives and for their entertainment.

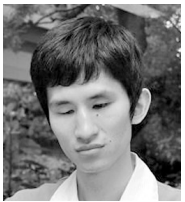

Kazunori Minatani finished a Doctor's program at the Graduate School of Political Studies, Gakushuin University in 2007. He was a Scientific Research Assistant at the National Institute of Special Needs Education in 2008 and a LimitedTerm Researcher at National Rehabilitation Center for the Disabled in 2009. Since October 2009, he has been an Associate Professor at the National Center for University Entrance Examinations. He has a Doctor's degree in Political Studies. 